\title{
Imaging findings and available percutaneous techniques for the treatment of bile leaks after hepatobiliary surgery
}

\author{
Salvatore Alessio Angileria, Giovanni Maria Rodàb, Anna Paola Savoldib, Letizia Di Megliob, \\ Giulia Signorellib, Anna Maria lerardia , Nikolaos Galanakisc, Dimitrios Tsetisc, Gianpaolo Carrafiello \\ Fondazione IRCCS Ca’ Granda Ospedale Maggiore Policlinico di Milano, Italy; Università Degli Studi di Milano, Italy; \\ University Hospital of Heraklion, Medical School, University of Crete, Greece
}

Abstract

${ }^{a}$ Operative Unit of Radiology, Fondazione IRCCS Ca' Granda Ospedale Maggiore Policlinico di Milano, Milano, Italy (Salvatore Alessio Angileri, Anna Maria Ierardi, Gianpaolo Carrafiello); 'Postgraduate School of Radiodiagnostics, Università Degli Studi di Milano, Milan, Italy (Giovanni Maria Rodà, Anna Paola Savoldi, Letizia Di Meglio, Giulia Signorelli); 'Department of Radiology, Interventional Radiology Unit, University Hospital of Heraklion, Medical School, University of Crete, Heraklion, Greece (Nikolaos Galanakis, Dimitrios Tsetis); dDepartment of Health Sciences, Università Degli Studi di Milano, Milan, Italy (Gianpaolo Carrafiello)

Conflict of Interest: None

Correspondence to: Salvatore Alessio Angileri, Operative Unit of Radiology, Fondazione IRCCS Ca' Granda Ospedale Maggiore Policlinico di Milano, Via Francesco Sforza 35, 20122, Milano, Italy, e-mail: ang.alessio@yahoo.it

Received 29 April 2020; accepted 6 July 2020; published online 16 September 2020

DOI: https://doi.org/10.20524/aog.2020.0532

\section{Introduction}

Bile leak is a common postoperative complication of surgical procedures for various hepatobiliary and pancreatic pathologies. The definition of postoperative bile leak remains arbitrary, with different cutoff values for drainage fluid volume and/or bilirubin concentration within various time intervals. This complication increases postoperative morbidity in already fragile patients and can lead to an increased risk of infections, prolonged hospital stays and need for reoperation [1].

The International Study Group of Liver Surgery (ISGLS) suggested a grading system based on the impact of this complication on the patients' clinical management [2].

- Bile leak grade A is the most common and has little or no impact on patients' management. The ISGLS suggests a non-operative management, based on close follow up with ultrasound (US), computed tomography $(\mathrm{CT})$, or magnetic resonance imaging and periodic blood examination evaluation. 
- Patients with grade B leaks present with a moderately compromised clinical condition. According to the ISGLS, endoscopic or percutaneous procedures are indicated in these patients.

- Patients with grade C leaks present with a severely compromised clinical condition. In these cases, the leak is due to complex duct transection or complete hepato-jejunal dehiscence with exclusion of liver segments. In this critical scenario, the ISGLS recommends a surgical approach [2].

Bile leak incidence is strictly linked with the type of surgery conducted, ranging from $0.9-9 \%$, with reported mortality rates between $8.7 \%$ and $39 \%$ and morbidity varying between $22 \%$ and $44 \%$ [3]. The appropriate diagnosis and treatment of postoperative biliary leaks are crucial to prevent further life-threatening complications, such as cholangitis, intraabdominal infection, biliary cirrhosis, end-stage liver disease and death [4-6]. The treatment of a bile leak is decided based on the severity and complexity of the leak. Surgical repair is rarely proposed in bile leaks classified as minor and in emergency situations, because of the notably high mortality rates following relaparotomy, ranging from $13-60 \%[7,8]$. In most cases, the options are endoscopic or radiological management [8].

Currently, interventional radiology (IR) is a rapidly expanding field that plays a pivotal role in the management of postoperative complications such as biliary leakage, with a growing number of applications as first-line therapy $[4,9,10]$. The aim of this study was to evaluate our experience in the management of postoperative bile leakages in the IR Unit, reporting the effectiveness of current percutaneous therapeutic techniques.

\section{Patients and methods}

This retrospective study was approved by the internal review board. Thirty-six patients (28 male, mean age: 68 years; and 8 female, mean age: 61 years), were treated for postoperative bile leak from January 2015 to May 2019. Both intrahepatic and extrahepatic leaks were treated (15 and 21, respectively). The type of treatment performed and relative percentage are reported in Table 1.

A biliary leak was clinically suspected in case of persistent leakage of bilious material from a surgical drain, or in the presence of non-specific symptoms such as abdominal pain, fever and anorexia, with or without alterations of liver enzymes on laboratory tests. Radiological confirmation was mainly based on noninvasive methods, such as US, CT and magnetic resonance cholangiopancreatography (MRCP) (Fig. 1,2), with or without hepato-specific contrast agents.

According to the ISGLS classification, the patients included in our study fell into 2 categories:

- Of 36 patients, 18 were first classified as having grade A bile leaks and treated with US or CT-guided percutaneous abdominal drainage (PAD) placement.

- The other 18 patients were classified as having grade B bile leaks. All grade B patients were treated with percutaneous transhepatic biliary drainage (PTBD) placement; 2 of them additionally underwent a percutaneous endoscopic
Table 1 Sex of patients, primary pathology and etiology of the leaks

\begin{tabular}{lcc} 
Parameters & $\begin{array}{c}\text { Number of } \\
\text { patients }\end{array}$ & $\begin{array}{c}\text { Percentage } \\
(\%)\end{array}$ \\
\hline Sex & 28 & 77.8 \\
$\quad$ Male (mean age 68 years) & 8 & 22.2 \\
Female (mean age 61 years) & & \\
Leak location & 15 & 41.6 \\
Intrahepatic & 21 & 58.4 \\
Extrahepatic & & \\
Primary pathology & 13 & 36.1 \\
Benign & 23 & 63.9 \\
Malignant & & \\
Treatment technique & 10 & 27.8 \\
Cholecystectomy without BEA & 6 & 16.7 \\
Cholecystectomy with BEA & 5 & 13.9 \\
Hepatic surgery without BEA & 4 & 11.1 \\
Hepatic surgery with BEA & 10 & 27.8 \\
Duodenopancreatectomy & 1 & 2.8 \\
Duodenectomy & 36 & 100 \\
Total & & \\
\hline BEA bilio-enteric anastomosis & &
\end{tabular}

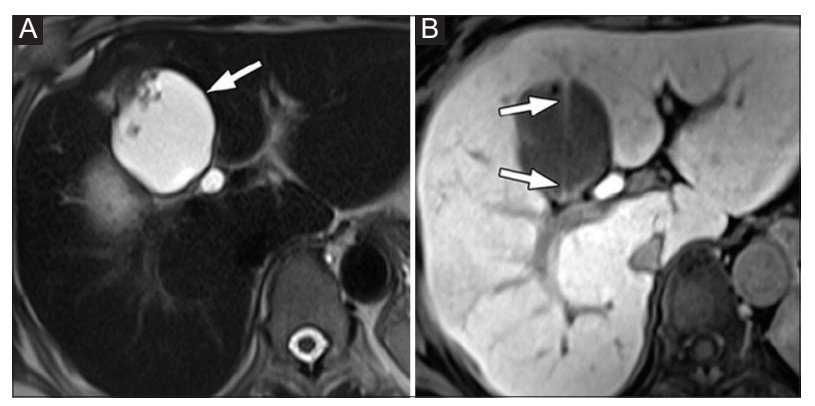

Figure 1 Bile leak after laparoscopic cholecystectomy in a 58-yearold woman. (A) Axial T2-weighted image obtained 45 days post intervention shows a loculated fluid collection (arrow) in the gallbladder bed. (B) Gd-EOB-DTPA-enhanced T1-weighted image obtained 60 min after contrast injection shows a jet of contrast material (arrows) within the loculated fluid collection, indicative of a bile leak

rendezvous (PE-RV) procedure, which combines an endoscopic and a percutaneous approach.

Percutaneous transhepatic cholangiography was performed in all candidates during the PTBD procedure to evaluate biliary anatomy, locate the site of the leak, and to determine the most suitable peripheral approach. In rare cases, endoscopic retrograde cholangiopancreatography was necessary in order to perform a PE-RV. Additional treatment actions, i.e., covered biliary stents and fistula embolization, were performed if the leak persisted.

We assessed the treatment effectiveness by evaluating several factors:

- Technical success (TS), defined as the ability to carry out the interventional procedure successfully. 

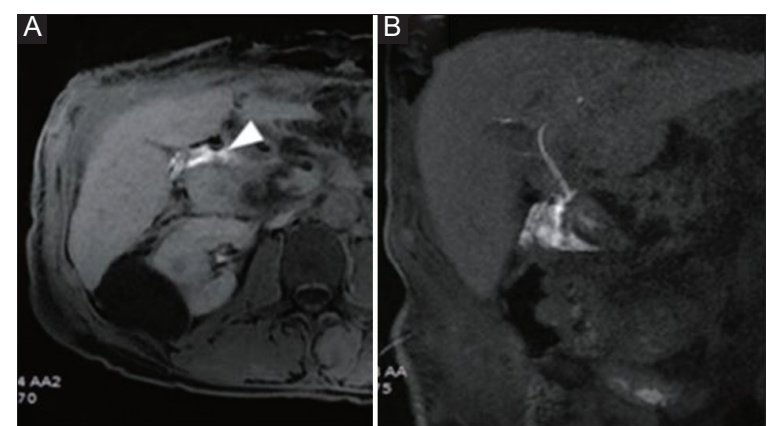

Figure 2 A 55-year-old man who underwent a Whipple procedure for pancreatic cancer and developed a biliary leakage from the biliodigestive anastomosis. (A) Axial post-contrast T1-weighted image showing hyperintense biloma (arrowhead); (B) Coronal post-contrast T1-weighted image showing extravasation of contrast material revealing the leakage location

- Clinical effectiveness (CE), defined as the ability to stop the leak. This was further classified into primary CE (PCE), when the leak was corrected with the first procedure alone, and secondary $\mathrm{CE}$ (SCE), when an additional procedure (e.g., stenting or embolization) was necessary in order to correct the leak. Overall CE (OCE) was achieved when the leak was controlled with either a single or multiple procedures.

Minor and major complications were evaluated according to the Society of Interventional Radiology guidelines [11].

\section{Results}

The success of the treatments is reported in the flowchart (Fig. 3). Of the 36 patients treated for bile leakage, 18 were initially classified as having grade A bile leakage and 18 as having grade B bile leakage. TS was calculated as $100 \%(36 / 36)$, as all IR procedures were carried out successfully.

All 18 patients with grade A leaks underwent PAD. The PCE of this procedure was $63.9 \%$ as 12 of 18 patients did not need additional treatment. However, 6 grade A bile leaks were later reclassified as grade B: of these, 1 patient was later treated with the PE-RV technique, while 5 patients underwent PTBD.

PTBD was also performed in all 18 patients originally classified as having grade B leaks. Of a total of 23 patients who underwent PTBD, as either first or second line of management, 12 received further treatment. In particular, 6 patients required further stent placement, 4 underwent fistula embolization and 2 were treated with the PE-RV technique. Of the 4 patients who required fistula embolization with plugs, coils and glue, 2 needed percutaneous stent placement in a later phase.

Of the total of 3 patients treated with the PE-RV technique, one later required percutaneous stent placement. A total of 9 patients required percutaneous placement of covered stents; of these, one required placement of 2 stents.

SCE was calculated as $36.1 \%$, as $1 / 18$ patients with grade A leaks and 12/18 patients with grade B leaks needed a second look and additional treatment. OCE was $100 \%$, as the final objective of leak healing was achieved in all patients who underwent interventional procedures.

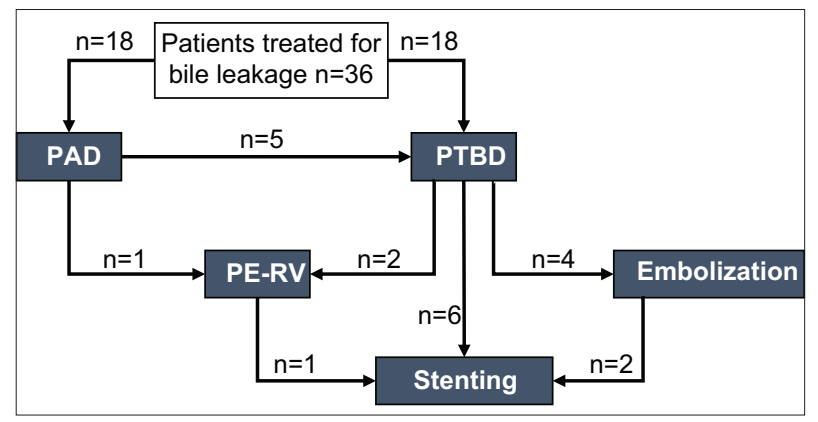

Figure 3 Patients' treatment success

$P A D$, percutaneous abdominal drainage; $P T B D$, percutaneous transhepatic biliary drainage; $P E-R V$, percutaneous-endoscopic rendezvous technique; $P C$ stenting, covered percutaneous stenting

There were no major complications immediately related to the interventional radiology procedures. Minor complications, such as fever, pain, bleeding from the point of access and pericatheter leakage, were observed in $7 / 36(19.4 \%)$ patients [12]. In particular 3 patients presented fever, 2 patients presented bleeding from the point of access, 1 patient complained about pain and 1 patient presented a peri-catheter leakage.

The drainage was kept for a mean of 14.7 days, ranging from a minimum of 7 to a maximum of 33 days. Hospital stay after the procedure varied from 9-41 days, with a mean of 18.5 days of stay. No procedure-related deaths occurred.

\section{Discussion}

Despite advances in surgical techniques and the perioperative management of hepatobiliary and pancreatic surgeries, biliary leaks remain a common complication that may lead to high morbidity and mortality rates. Surgical relaparotomy is a difficult procedure that requires high surgical competence and shows high mortality rates.

In our center, biliary leaks are managed between the IR and Endoscopy teams. More specifically, the intrahepatic leaks undergo an IR treatment while the extrahepatic ones can be managed by both IR and Endoscopy; they thus require a multidisciplinary evaluation in order to choose the most suitable treatment for each patient. In our case, 15/36 leaks were intrahepatic; thus those patients were directly managed using IR. Among the 21/36 extrahepatic leaks, in 11 there was a hepatojejunostomy so the IR approach was mandatory. In the other 10 cases, treatment was determined by the multidisciplinary evaluation [13]. Percutaneous IR procedures are preferred as a first-line therapeutic option to avoid more invasive procedures. The role of IR is to diagnose biliary damage and to treat it either with a definitive curative purpose, or as a bridge to relaparotomy.

The diagnosis of bile leakage was achieved in most patients using noninvasive methods, i.e., US, CT and MRCP. Its treatment depends on the severity of the leak (grades A-C), taking into account the impact on the patient's clinical management $[5,14,15]$. To allow healing of the biliary lesion, it is essential to drain the bile collections to avoid or treat superinfections and to restore the exclusive continuity between the biliary tract and the 
digestive system. PAD, PTBD, and PE-RV are safe and effective procedures for the treatment of postsurgical biliary leaks.

Grade A bile leaks have little or no impact on clinical management, since they have a spontaneous resolution, otherwise they can be controlled by intra-abdominal drainage, placed during the surgical intervention or in a separate radiological intervention session, with a persistent decrease in volume of drain fluid and bilirubin concentration in the fluid. Additional diagnostic or therapeutic interventions are usually not necessary [14-16].

In our cases, TS represented $100 \%$, as all interventional percutaneous procedures were carried out successfully. Our results are consistent with the literature, which reports a success rate for CT-guided PAD ranging from $75.7-86.9 \%$ up to $100 \%$, with only a few patients requiring more than one drainage $[5,9]$.

In the case of grade B bile leaks, interventional radiologic and endoscopic procedures are often suggested by ISGLS along with antibiotic therapy $[6,13]$. The aim is to create a low-pressure system along the biliary tract by redirecting the bile flow from the site of the defect into the bile ducts and duodenum. These procedures may also be used as a bridging therapy to stabilize the patient before surgery in the case of grade $\mathrm{C}$ bile leaks $[5,9,17]$.

TS rates of PTBD for biliary leak are reported to be 40 $100 \%$ without secondary surgery (Table 2) $[5,8,18,19]$. The wide range of success rates is attributable to the use of different interventional strategies (Fig. 4) [20]. Our data for PTBD procedures are consistent with the literature.

If the biliary leak persists, other safe and effective interventional procedures may be considered, depending on the characteristics of the bile injury. In particular, a PE-RV procedure can be performed with the aid of endoscopy, or the isolated biliary duct may be occluded using embolizing agents, such as fibrin, acetic acid, ethanol, and glues. Covered stents, coils or plugs can be used to embolize leaks or fistulas communicating with the biliary tree (Fig. 5,6) [5].

In our case, the calculated PCE for all patients was 63.9\%, suggesting that the condition may be solved using the firstchoice procedure. SCE was calculated as $36.1 \%$; the rather low percentage was expected and consistent with the literature, as only a few $(n=16)$ patients required a second look with IR procedures. None of these patients required traditional surgery.

Grade C bile leaks usually require relaparotomy. Operative procedures may include maneuvers such as suture closure

Table 2 Biliary leaks treated with interventional radiology procedures and success rate

\begin{tabular}{lcccl}
\hline Authors & $\mathrm{N}$ & Total & $\%$ & Technique \\
\hline Popat et al, 2017 [19] & 62 & 157 & 40 & PTBD \\
Cozzi et al, 2006 [21] & 15 & 17 & 88 & PTBD \\
Mastier et al, , 2018 [8] & 75 & 101 & 74 & PTBD \\
Pedicini et al, $2010[22]$ & 11 & 16 & 68 & PTBD \\
Aytekin et al,, 2007 [23] & 10 & 10 & 100 & PTBD \\
Righi et al,, 2008 [24] & 21 & 23 & 91 & PTBD \\
Sohn et al, , 2003 [25] & 32 & 39 & 82 & PTC/PTBD \\
\hline
\end{tabular}

$\mathrm{N}^{\circ}$, number of successes; Total, number of patients treated with interventional radiology procedures

PTC, percutaneous transhepatic cholangiography; PTBD, percutaneous transhepatic biliary drainage

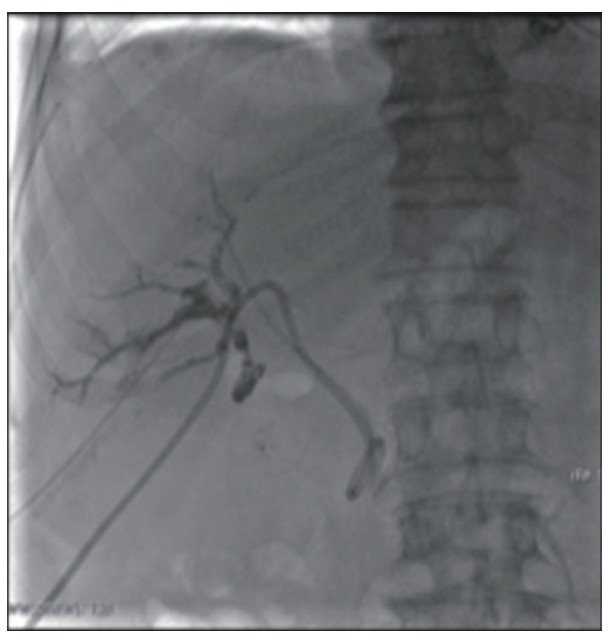

Figure 4 Right-sided percutaneous transhepatic biliary drainage. Percutaneous transhepatic cholangiography during the procedure demonstrating the presence of biliary leakage (arrow)
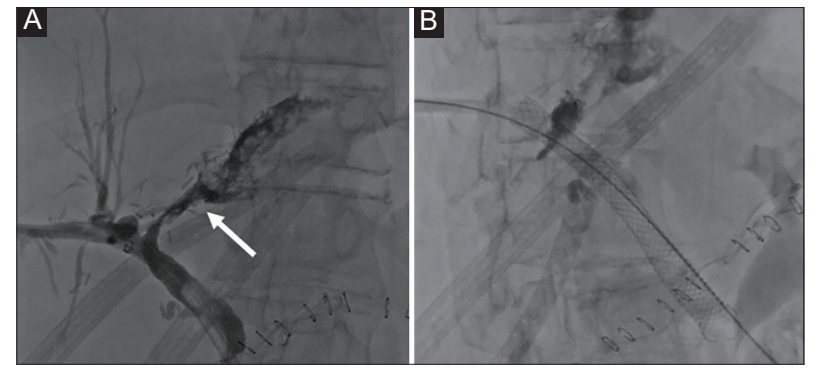

Figure 5 Biliary leak. (A) Percutaneous transhepatic cholangiography demonstrated biliary leakage (arrow) after hepatectomy; (B) stent and plug placement successfully covered the leakage.
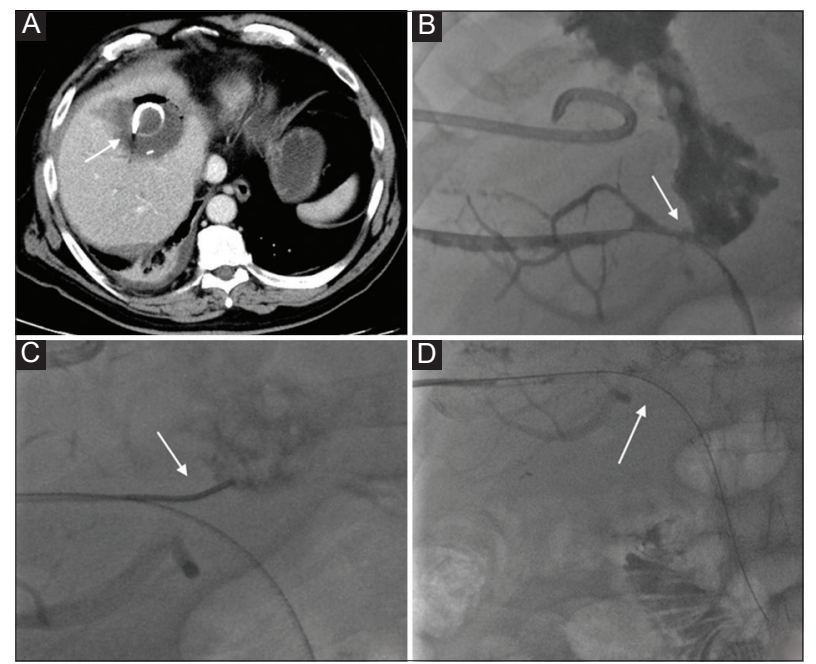

Figure 6 Patient with intrahepatic leak after a left hepatectomy. Axial view contrast enhanced computed tomography scan showing a biloma with percutaneous abdominal drainage within it (white arrow) (A); cholangiography showing the leak's origin refilling the biloma with the drainage within it (B); during the procedure the catheter has been positioned at the leak's origin to perform an embolization with glue (C); final step of the procedure: a covered stent has been positioned to cover the leak's origin (D) 
of leaking bile ducts, clearance of intra-abdominal fluid collections, reconstruction of a bilio-enteric anastomosis and placement of additional drains for continuous postoperative lavage $[14,15]$. No grade $C$ leak was observed among our cases.

The limitations of our retrospective study are the relatively short time frame taken into consideration (January 2015-May 2019) and, as a consequence, the number of patients included in the study. Another limitation is the lack of data regarding some of the patients' outcomes.

In conclusion, considering all percutaneous techniques, in our study the OCE and therefore leak healing was achieved in all the patients with grade A and B biliary leaks. These procedures provide a less invasive approach, are increasingly recognized as having a significant role in the management of complications, and should be considered an integral component in the postoperative management of these patients.

\section{Summary Box}

\section{What is already known:}

- Bile leak is a common postoperative complication of surgical procedures for various hepatobiliary and pancreatic pathologies

- Appropriate diagnosis and treatment of postoperative biliary leaks are crucial to prevent further life-threatening complications

- In the case of high-grade bile leaks, interventional radiologic and endoscopic procedures are often performed along with antibiotic therapy, as suggested by International Study Group of Liver Surgery

\section{What the new findings are:}

- In our study the overall clinical effectiveness and therefore leak healing was achieved in all the patients with grade A and B biliary leaks

- Procedures used in our interventional radiology Unit provide a less invasive approach and are increasingly recognized as having a significant role in the management of complications

- Our calculated primary clinical effectiveness for all patients suggests that the bile leakage may be solved using the first-choice procedure

\section{References}

1. Clavien PA, Barkun J, de Oliveira ML, et al. The Clavien-Dindo classification of surgical complications: five-year experience. Ann Surg 2009;250:187-196.

2. Dindo D, Demartines N, Clavien PA. Classification of surgical complications: a new proposal with evaluation in a cohort of 6336 patients and results of a survey. Ann Surg 2004;240:205-213.

3. Petrillo M, Ierardi AM, Tofanelli L, et al. Gd-EOB-DTP-enhanced MRC in the preoperative percutaneous management of intra and extrahepatic biliary leakages: does it matter? Gland Surg 2019;8:174-183.

4. Angileri SA, Gorga G, Tortora S, et al. Biliary injuries after pancreatic surgery: interventional radiology management. Gland Surg 2019;8:141-149.

5. Slanetz PJ, Boland GW, Mueller PR. Imaging and interventional radiology in laparoscopic injuries to the gallbladder and biliary system. Radiology 1996;201:595-603.

6. Yun SU, Cheon YK, Shim CS, et al. The outcome of endoscopic management of bile leakage after hepatobiliary surgery. Korean $J$ Intern Med 2017;32:79-84.

7. Casadei R, Ricci C, Giampalma E, et al. Interventional radiology procedures after pancreatic resections for pancreatic and periampullary diseases. JOP 2014;15:378-382.

8. Mastier C, Valette PJ, Adham M, et al. Complex biliaryleaks: effectiveness of percutaneous radiological treatment compared to simple leaks in 101 patients. Cardiovasc Intervent Radiol 2018;41:1566-1572.

9. May K, Hunold P. Leakage of hepaticojejunal anastomosis: radiological interventional therapy. Visc Med 2017;33:192-196.

10. Baker TA, Aaron JM, Borge M, Pierce K, Shoup M, Aranha GV. Role of interventional radiology in the management of complications after pancreaticoduodenectomy. Am J Surg 2008;195:386-390.

11. Khalilzadeh O, Baerlocher MO, Shyn PB, et al. Proposal of a new adverse event classification by the Society of Interventional Radiology Standards of Practice Committee. J Vasc Interv Radiol 2017;28:1432-1437.e3.

12. May K, Hunold P. Leakage of hepaticojejunal anastomosis: radiological interventional therapy. Visc Med 2017;33:192-196.

13. Hoekstra LT, van GulikTM, Gouma DJ, Busch OR. Posthepatectomy bile leakage: how to manage. Dig Surg 2012;29:48-53.

14. Koch M, Garden OJ, Padbury R, et al. Bile leakage after hepatobiliary and pancreatic surgery: a definition and grading of severity by the International Study Group of Liver Surgery. Surgery 2011;149:680-688.

15. Panaro F, Hacina L, Bouyabrine H, Al-Hashmi AW, Herrero A, Navarro F. Risk factors for postoperative bile leakage: a retrospective single-center analysis of 411 hepatectomies. Hepatobiliary Pancreat Dis Int 2016;15:81-86.

16. Malgras B, Duron S, Gaujoux S, et al. Early biliary complications following pancreaticoduodenectomy: prevalence and risk factors. HPB (Oxford) 2016;18:367-374.

17. Sabry AA, El Fouly MG, Fawzy MWED. Leak after laparoscopic cholecystectomy; incidence and management. Egypt J Hosp Med 2018;72:5093-5098.

18. Sohn TA, Yeo CJ, Cameron JL, et al. Pancreaticoduodenectomy: role of interventional radiologists in managing patients and complications. J Gastrointest Surg 2003;7:209-219.

19. Popat B, Thakkar D, Deshmukh H, Rathod K. Percutaneous transhepatic biliary drainage in the management of post-surgical biliary leaks. Indian J Surg 2017;79:24-28.

20. Kühn JP, Busemann A, Lerch MM, Heidecke CD, Hosten N, Puls R. Percutaneous biliary drainage in patients with nondilated intrahepatic bile ducts compared with patients with dilated intrahepatic bile ducts. AJR Am J Roentgenol 2010;195:851-857.

21. Cozzi G, Severini A, Civelli E, et al. Percutaneous transhepatic biliary drainage in the management of postsurgical biliary leaks in patients with nondilated intrahepatic bile ducts. Cardiovasc Intervent Radiol 2006;29:380-388.

22. Pedicini V, Poretti D, Mauri G, et al. Management of post-surgical biliary leakage with percutaneous transhepatic biliary drainage (PTBD) and occlusion balloon (OB) in patients without dilatation of the biliary tree: preliminary results. Eur Radiol 2010;20:1061-1068.

23. Aytekin C, Boyvat F, Harman A, Ozyer U, Sevmiş S, Haberal M. Percutaneous management of anastomotic bile leaks following liver transplantation. Diagn Interv Radiol 2007;13:101-104.

24. Righi D, Franchello A, Ricchiuti A, et al. Safety and efficacy of the percutaneous treatment of bile leaks in hepaticojejunostomy or split-liver transplantation without dilatation of the biliary tree. Liver Transpl 2008;14:611-615.

25. Sohn TA, Yeo CJ, Cameron JL, et al. Pancreaticoduodenectomy: role of interventional radiologists in managing patients and complications. J Gastrointest Surg 2003;7:209-219. 